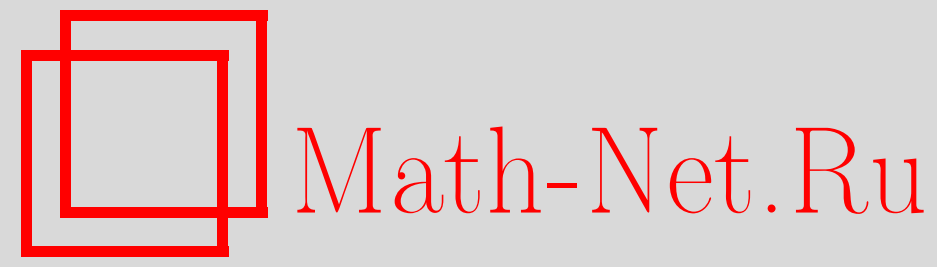

В. А. Коваль, О законе повторного логарифма для операторно-нормированных сумм случайных векторов, Теория вероятн. и ее примен., 2001, том 46, выпуск 2, 381-383

DOI: https://doi.org/10.4213/tvp3927

Использование Общероссийского математического портала MathNet.Ru подразумевает, что вы прочитали и согласны с пользовательским соглашением

http://www . mathnet.ru/rus/agreement

Параметры загрузки:

IP : 52.6 .47 .48

26 апреля 2023 г., 15:27:38

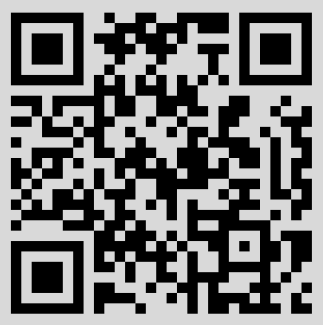


11. Риехстыньи Э.Я. Асимптотические разложения интегралов. Т. 2. Рига: Зинатне, 1977,464 с.

\section{О ЗАКОНЕ ПОВТОРНОГО ЛОГАРИФМА ДЛЯ ОПЕРАТОРНО-НОРМИРОВАННЫХ СУММ СЛУЧАЙНЫХ ВЕКТОРОВ}

Доказывается закон повторного логарифма для сумм случайных векторов в $\mathbf{R}^{k}$, нормированных линейными операторами общего вида. При этом предполагается, что последовательность случайных векторов удовлетворяет принципу инвариантности Штрассена.

Ключевые слова и фразы: закон повторного логарифма, почти наверное принци инвариантности, операторно-нормированные суммы случайных векторов.

Пусть $\left(A_{n}, n \geqslant 1\right)$ - последовательность линейных операторов, действующих из $\mathbf{R}^{k}$ в $\mathbf{R}^{l}(1 \leqslant k, l<\infty) ;\left(X_{n}, n \geqslant 1\right)$ - последовательность случайных векторов в $\mathbf{R}^{k} ;\|x\|$ - евклидова норма вектора $x \in \mathbf{R}^{k}\left(\mathbf{R}^{l}\right)$. Для оператора $B$, действуюшего из $\mathbf{R}^{k}$ в $\mathbf{R}^{l}\left(\mathbf{R}^{k}\right)$, полагаем $\|B\|=\sup _{\|x\|=1}\|B x\|$. Закон повторного логарифма (3ПЛ) для операторно-нормированных сумм $\left\|A_{n} \sum_{i=1}^{n} X_{i}\right\|$ при определенных ограничениях на операторы $\left(A_{n}, n \geqslant 1\right)$ изучался в работах [1], [2]. В [3] исследовался аналог ограниченного 3ПЛ в случае общих условий на $\left(A_{n}, n \geqslant 1\right)$. Мы докажем 3ПЛ, ограничившись лишь одним естественным требованием к $\left(A_{n}, n \geqslant 1\right)$, и при этом будем предполагать, что последовательность $\left(X_{n}, n \geqslant 1\right)$ удовлетворяет принципу инвариантности Штрассена [4], что позволяет охватить достаточно широкий класс последовательностей $\left(X_{n}, n \geqslant 1\right)$.

Отметим также, что усиленные законы больших чисел с операторными нормировками исследовались в работах [3], [5], [6].

Теорема. Пусть случайкье векторы $\left(X_{n}, n \geqslant 1\right)$ чентрировакъ, имект обиий ковариачиояныน̆ оператор $D(D \neq 0)$ и удовлетворяют следуючему условию: существует последователькость кезависимых чентрированкых одияаково распределенкых гауссовских случайкых векторов $\left(\Gamma_{n}, n \geqslant 1\right)$ в $\mathbf{R}^{k}$ с ковариачиокным оператором $D$ maxux, чmo

$$
\left\|\sum_{i=1}^{n} X_{i}-\sum_{i=1}^{n} \Gamma_{i}\right\|=o\left((n \ln \ln n)^{1 / 2}\right) \quad \text { п.к., } n \rightarrow \infty .
$$

Eсли $\left\|A_{n} D^{1 / 2}\right\| \neq 0$ при всех $n \geqslant n_{0} \geqslant 1\left(D^{1 / 2}-\right.$ квадраткьй корекь из $\left.D\right)$, то

$$
\limsup _{n \rightarrow \infty} \frac{\left\|A_{n} \sum_{i=1}^{n} X_{i}\right\|}{\left\|A_{n} D^{1 / 2}\right\|(2 n \ln \ln n)^{1 / 2}}=1 \quad \text { n.н. }
$$

3 а м е ч а н и е 1. Соотношение (1), называемое также «почти наверное принципом инвариантности», впервые было установлено в работе [4] для независимых центрированных одинаково распределенных случайных величин с конечной дисперсией. Его обобщение на многомерный случай было получено в [7]. Отметим, что, кроме последовательностей независимых центрированных одинаково распределенных случайных векторов $\left(X_{n}, n \geqslant 1\right)$ с $\mathbf{E}\left\|X_{n}\right\|^{2}<\infty$, соотношению (1) удовлетворяют:

* Национальный технический университет Украины, кафедра высшей математики, просп. Победы, 37, 252056 Киев, Украина; e-mail: luyda@net.zt.ua 
стационарные эргодические мартингал-разности $\left(X_{n}, n \geqslant 1\right)$ с конечным вторым моментом, стационарные последовательности с различными условиями перемешивания и функции от них (см. [8]), мартингалы и последовательности независимых центрированных случайных векторов $\left(X_{n}, n \geqslant 1\right)$ с общим ковариационным оператором при $\mathbf{E}\left\|X_{n}\right\|^{2+\delta}<\infty, n \geqslant 1$ (см. [9]).

3 а м е ч а и е 2 . Если $\left\|A_{n}\right\| \neq 0$ и существует обратный оператор $D^{-1}$, то $\left\|A_{n} D^{1 / 2}\right\| \neq 0$.

Д ок а 3 а т е л ь с т о т е о р е мы. 1) Предположим сначала, что для оператора $D$ существует обратный оператор $D^{-1}$. Положим $a_{n}=(2 n \ln \ln n)^{1 / 2}, n \geqslant 3$; $c_{n}=\left\|A_{n} D^{1 / 2}\right\| a_{n}, n \geqslant 3 ; Y_{n}=\sum_{i=1}^{n} \Gamma_{i}, n \geqslant 1$. Так как

$$
\begin{aligned}
& \left|\limsup _{n \rightarrow \infty} c_{n}^{-1}\left\|A_{n} \sum_{i=1}^{n} X_{i}\right\|-\limsup _{n \rightarrow \infty} c_{n}^{-1}\left\|A_{n} Y_{n}\right\|\right| \\
& \leqslant\left\|D^{-1 / 2}\right\| \underset{n \rightarrow \infty}{\limsup a_{n}^{-1} \|}\left\|\sum_{i=1}^{n} X_{i}-Y_{n}\right\|=0 \text { п.н., }
\end{aligned}
$$

то для доказательства теоремы нужно показать, что $\lim \sup _{n \rightarrow \infty} c_{n}^{-1}\left\|A_{n} Y_{n}\right\|=1$ п.н. Очевидно, что достаточно рассмотреть случай, когда $D$ есть тождественный оператор.

Так как в силу [10] $\lim \sup _{n \rightarrow \infty}\left[\left\|A_{n} Y_{n}\right\| /\left(\left\|A_{n}\right\| a_{n}\right)\right] \leqslant 1$ п.н., то для доказательства теоремы нужно показать, что

$$
\limsup _{n \rightarrow \infty} \frac{\left\|A_{n} Y_{n}\right\|}{\left\|A_{n}\right\| a_{n}} \geqslant 1 \text { п.н. }
$$

Докажем (2) сначала в случае, когда $A_{n} Y_{n}=\left\langle T_{n}, Y_{n}\right\rangle$, где $\left(T_{n}, n \geqslant 1\right)$ - последовательность векторов в $\mathbf{R}^{k}$ таких, что $\left\|T_{n}\right\| \neq 0, n \geqslant n_{0} \geqslant 1 ;\langle\cdot, \cdot\rangle$ - скалярное произведение в $\mathbf{R}^{k}$. То есть покажем, что

$$
\limsup _{n \rightarrow \infty} \frac{\left\langle T_{n}, Y_{n}\right\rangle}{\left\|T_{n}\right\| a_{n}} \geqslant 1 \text { п.н. }
$$

Положим $\xi_{n}=\left\langle U_{n}, Y_{n}\right\rangle, n \geqslant 1$, где $U_{n}=T_{n} /\left\|T_{n}\right\| . \quad\left(\xi_{n}, n \geqslant 1\right)$ есть гауссовская последовательность в $\mathbf{R}^{1}$ с нулевым средним. Заметим, что $\sigma_{n}^{2}=\mathbf{E} \xi_{n}^{2}=n$ и при всех $\boldsymbol{m}<\boldsymbol{n}$

$$
r_{m, n}=\frac{\mathbf{E}\left(\xi_{m} \xi_{n}\right)}{\sigma_{m} \sigma_{n}}=\frac{m^{1 / 2}\left\langle U_{m}, U_{n}\right\rangle}{n^{1 / 2}} \leqslant\left(\frac{m}{n}\right)^{1 / 2} .
$$

Рассмотрим последовательность независимых гауссовских $\mathscr{N}(0,1)$-распределенных случайных величин $\left(\gamma_{n}, n \geqslant 1\right)$. ПІоложим $\tilde{\xi}_{n}=\sum_{i=1}^{n} \gamma_{i}, n \geqslant 1$. При этом заметим, что $\tilde{\sigma}_{n}^{2}=\mathbf{E} \tilde{\xi}_{n}^{2}=\mathbf{E} \xi_{n}^{2}, n \geqslant 1$, и $\tilde{r}_{m, n}=\left(\tilde{\sigma}_{m} \tilde{\sigma}_{n}\right)^{-1} \mathbf{E}\left(\tilde{\xi}_{m} \tilde{\xi}_{n}\right) \geqslant r_{m, n}$ при всех $m<n$ в силу (4). Поэтому, согласно неравенству Слепяна [11] и ЗПЛ Хартмана-Винтнера,

$$
\limsup _{n \rightarrow \infty} a_{n}^{-1} \xi_{n} \geqslant \limsup _{n \rightarrow \infty} a_{n}^{-1} \tilde{\xi}_{n}=1 \quad \text { п.н. }
$$

Таким образом, соотношение (3) доказано.

Докажем (2) в общем случае. Найдется последовательность векторов $\left(e_{n}, n \geqslant 1\right)$ в $\mathbf{R}^{\prime}$ с $\left\|e_{n}\right\|=1$ таких, что $\left\|A_{n}^{*} e_{n}\right\|=\left\|A_{n}^{*}\right\|$ (см., например, [12]), где $A_{n}^{*}$ - оператор, сопряженный $A_{n}$. Поэтому (п.н.)

$$
\limsup _{n \rightarrow \infty} \frac{\left\|A_{n} Y_{n}\right\|}{\left\|A_{n}\right\| a_{n}} \geqslant \limsup _{n \rightarrow \infty} \frac{\left|\left\langle e_{n}, A_{n} Y_{n}\right\rangle\right|}{\left\|A_{n}^{*}\right\| a_{n}}=\limsup _{n \rightarrow \infty} \frac{\left|\left\langle A_{n}^{*} e_{n}, Y_{n}\right\rangle\right|}{\left\|A_{n}^{*} e_{n}\right\| a_{n}} \geqslant 1
$$

в силу (3).

Таким образом, если существует обратный оператор $D^{-1}$, то теорема доказана.

2) Предположим, что оператор $D$ вырожденный. Рассмотрим подпространство $D^{1 / 2} \mathbf{R}^{k}$ пространства $\mathbf{R}^{k}$. На этом подпространстве определен обратный оператор $D^{-1}$ и в силу уже доказанного

$$
\limsup _{n \rightarrow \infty} \frac{\left\|A_{n} \sum_{i=1}^{n} X_{i}\right\|}{\left\|A_{n} D^{1 / 2}\right\|_{*} a_{n}}=1 \text { п.н., }
$$


где $\left\|A_{n} D^{1 / 2}\right\|_{*}=\sup _{x \in D^{1 / 2} \mathbf{R}^{k}, x \neq 0}\left(\left\|A_{n} D^{1 / 2} x\right\| /\|x\|\right)$. Нетрудно убедиться, что $\left\|A_{n} D^{1 / 2}\right\|_{*}=\left\|A_{n} D^{1 / 2}\right\|$. Теорема доказана.

Автор выражает благодарность профессору В.В. Булдыгину за внимание к работе и полезные советы.

\section{СПИСОК ЛИТЕРАТУРЫ}

1. Weiner D.C. A law of the iterated logarithm for distributions in the generalized domain of attraction of a nondegenerate Gaussian law. - Probab. Theory Relat. Fields, 1986, v. 72, № 3, p. 337-357.

2. Lai T.L. Some almost sure convergence properties of weighted sums of martingale difference sequences. - Almost Everywhere Convergence II: Proceedings of the 2nd International Conference, Evanston, IL, 1989. Boston: Academic Press, 1991, p. 179190.

3. Buldygin V., Solntsev S. Asymptotic behaviour of linearly transformed sums of random variables. Dordrecht: Kluwer, 1997, 516 p.

4. Strassen $V$. An invariance principle for the law of the iterated logarithm. - Z Wahrscheinlich keitstheor. verw. Geb., 1964, B. 3, № 3, S. 211-226.

5. Мельяиков A.B. Закон больших чисел для многомерных мартингалов. - Докл. AH CCCP, 1986, т. 286, № 3, c. 546-550.

6. Kaufmann $H$. On the strong law of large numbers for multivariate martingales. Stochastic Process. Appl., 1987, v. 26, № 1, p. 73-85.

7. Philipp $W$. Almost sure invariance principles for sums of $B$-valued random variables. - Lecture Notes in Math., 1979, v. 709, p. 171-193.

8. Berger $E$. An almost sure invariance principle for stationary ergodic sequences of Banach space valued random variables. - Probab. Theory Relat. Fields, 1990, v. 84, № 2, p. 161-201.

9. Morrow G., Philipp W. An almost sure invariance principle for Hilbert space valued martingales. - Trans. Amer. Math. Soc., 1982, v. 273, № 1, p. 231-251.

10. Finkelstein $H$. The law of the iterated logarithm for empirical distributions. - Ann. Math. Statist., 1971, v. 42, № 2, p. 607-615.

11. Slepian D. The one-sided barrier problem for Gaussian noise. - Bell Syst. Tech. J., 1962 , v. 41 , p. $463-501$.

12. Воеводия В.В., Кузнецов Ю. А. Матрицы и вычисления. М.: Наука, 1984, 320 с.

Поступила в редакцию

9.VIII.1999

(C) $2001 \Gamma$.

КОНДРАТЕНКО А. Е.*

\section{СВЯЗЬ СКОРОСТИ СХОДИМОСТИ МОМЕНТОВ НОРМИРОВАННЫХ СУММ С МОМЕНТАМИ ЧЕБЫIIЕВА-ЭРМИТА}

Представлека акад. Ю.В. Прохоровьмм

В работе получено выражение обычных моментов через моменты Чебышева-Эрмита. Этот результат используется в задаче о скорости сходимости моментов в центральной предельной теореме.

Ключевые слова и фразы: центральная предельная теорема, скорость сходимости, моменты, моменты Чебышева-Эрмита.

* Московский государственный университет им. М. В. Ломоносова, механикоматематический факультет, кафедра теории вероятностей, Воробьевы горы, 119899 Москва, Россия; e-mail: a_cond@mail.ru 